\title{
Critical calcified carotid stenosis treated with shockwave lithoplasty
}

Placido Grillo, Cesare Tripolino, Eliezer Joseph Tassone, Gaetano Morabito, Bindo Missiroli

Cardiology Unit, Sant'Anna Hospital, Catanzaro, Italy

Submitted: 11 September 2018

Accepted: 5 November 2018

Arch Med Sci Atheroscler Dis 2018; 3: e164-e165 DOI: https://doi.org/10.5114/amsad.2018.81010 Copyright @ 2018 Termedia \& Banach

Despite many improvements in endovascular techniques, the treatment of vascular calcified stenosis remains a great challenge for the cardiologist. Recently a new technology called "Shockwave Lithoplasty System" has been introduced, and it seems able to break calcium deposits without affecting the soft tissues [1-3]. A 50-year-old man with diabetes and hypertension came to our observation for the diagnosis of bilateral critical stenosis of the internal carotid arteries (ICAs). Of note, he had undergone previous radiotherapy at the neck level for a tongue carcinoma. Surgical treatment was deemed risky and consequently percutaneous transluminal angioplasty (PTA) was chosen as the method of revascularization [4]. Selective angiography showed the presence of critical stenosis of the proximal segment of the left ICA due to a fibrolipidic plaque and a double critical stenosis of the proximal segment of the right ICA due to a calcific plaque (Figure $1 \mathrm{~A}$ ). The left ICA was successful treated with PTA and stenting. However, the calcified nature of the right ICA made endovascular treatment very difficult with the common devices. For this reason, the patient was readmitted one month later to undergo Shockwave Lithoplasty. Briefly, under local anesthesia the left femoral artery was cannulated with a 6 -French sheath. After positioning an embolic protection device (SpiderFX, Medtronic), a $4.0 \times 15 \mathrm{~mm}$ Lithoplasty balloon (Shockwave C2 ivL, Shockwave Medical) was applied at the lesion level. It was inflated at $4 \mathrm{~atm}$ and 5 pulses of ultrasound energy of $10 \mathrm{~s}$ were applied. The balloon was then inflated to $6 \mathrm{~atm}$ for about $15 \mathrm{~s}$. Once the Lithoplasty treatment was finished, a $9 \times 40 \mathrm{~mm}$ self-expanding stent (Carotid Wall Stent, Boston Scientific) was deployed and post dilated with a $5.5 \times 15 \mathrm{~mm}$ balloon (Maverick XL, Boston Scientific) at $12 \mathrm{~atm}$ and with a $4.5 \times 15 \mathrm{~mm}$ balloon (Maverick XL, Boston Scientific) at $14 \mathrm{~atm}$. Final angiography demonstrated excellent position of the stent, good wall apposition, and confirmed patency of the right carotid artery (Figure 1 B).

The Lithoplasty system is a novel technology that integrates angioplasty balloon catheter devices with the calcium-disrupting power of sonic pressure waves. Each Lithoplasty catheter incorporates multiple lithotripsy emitters activated with the touch of a button after the balloon is inflated. Once activated, these emitters produce sonic pressure waves that are highly tissue-selective, passing through the balloon and soft vascular tissue, to selectively disrupt calcium. Once the calcium has been modified, the vessel can be dilated using low pressures. The Lithoplasty system received FDA approval in 2016, and has been available in Europe since 2015. To date, it is employed for the treatment of calcified lesions in coronary, iliac, and infra-popliteal arteries [5]. Safety and performance of Lithoplasty

\author{
Corresponding author: \\ Dr. Cesare Tripolino \\ Cardiology Unit \\ Sant'Anna Hospital \\ Viale Pio $X$ \\ 88100 Catanzaro, Italy \\ Phone: +3909650701 \\ E-mail: cesare.tripolino@ \\ virgilio.it
}



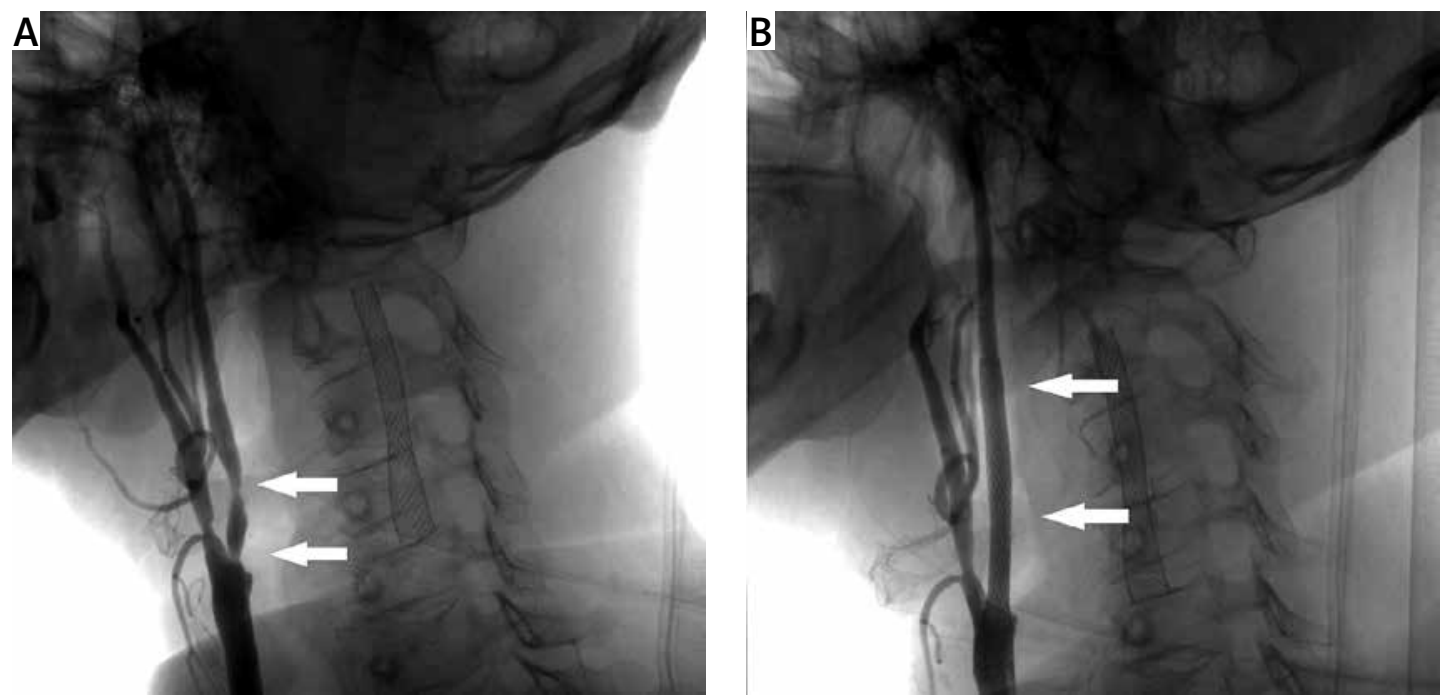

Figure 1. Angiography showing: critical calcified plaque of right internal carotid (arrows) (A); right internal carotid appearance after stenting (white arrows) (B)

are supported by the results of DISRUPT PAD (I and II) trials. These are prospective multi-center, single-arm studies carried out in patients with moderate to severely calcified femoro-popliteal lesions. The results of these trials demonstrated the safety of the Lithoplasty system with minimal vessel injury, and high procedural success with a large gain in arterial diameter. Thirty-day patency assessed by duplex ultrasound was $100 \%$, and at 6 months arterial patency was $81 \%$ [6].

Similarly, the DISRUPT CAD study demonstrated high luminal acute gain independent of the degree of calcification, in patients with heavily calcified coronary stenosis. Among these, 31 patients underwent OCT before and after Lithoplasty treatment (DISRUPT CAD OCT sub-study). High-resolution imaging delineated calcium modification with fracture as a major mechanism of action of Lithoplasty [1]. In addition, while other devices (i.e. rotational atherectomy) generate microparticles that embolize distally, large calcium fragments generated by Lithoplasty seem to remain in situ, not impairing the microcirculation.

Interestingly, in a quite recent paper, Gorla et al. described the case of an 87-year-old woman with aortic stenosis in whom transcatheter aortic valve implantation through the transfemoral approach was a challenge due to calcified lesions of iliac artery [7]. After treatment with the Lithoplasty system, the authors were able to overcome the calcified lesion, thus allowing transfemoral transcatheter aortic valve implantation (TAVI) to be performed. In this scenario, Lithoplasty may help to expand eligibility for transfemoral TAVI to patients with calcific ileo-femoral vessels and at high risk for a non-transfemoral approach.

In our case, we employed Lithoplasty to treat heavily calcified plaques in a patient in whom endarterectomy would be difficult and risky due to the previous radiotherapy treatment. Our experience demonstrates that Lithoplasty might be a new and effective tool for the management of calcified stenosis, including the carotid district, allowing better stent delivery and expansion.

\section{Conflict of interest}

The authors declare no conflict of interest.

\section{References}

1. Ali ZA, Brinton TJ, Hill JM, et al. Optical coherence tomography characterization of coronary lithoplasty for treatment of calcified lesions: first description. JACC Cardiovasc Imaging 2017; 10: 897-906.

2. De Silva K, Roy J, Webb I, et al. A calcific, undilatable stenosis: lithoplasty, a new tool in the box? JACC Cardiovasc Interv 2017; 10: 304-6.

3. Brodmann M, Holden A, Zeller T. Safety and feasibility of intravascular lithotripsy for treatment of below-theknee arterial stenoses. J Endovasc Ther 2018; 25: 499503.

4. Harrod-Kim P, Kadkhodayan Y, Derdeyn CP, Cross DT 3rd, Moran CJ. Outcomes of carotid angioplasty and stenting for radiation-associated stenosis. AJNR Am J Neuroradiol 2005; 26: 1781-8.

5. Brodmann $M$, Werner $M$, Brinton TJ, et al. Safety and performance of lithoplasty for treatment of calcified peripheral artery lesions. J Am Coll Cardiol 2017; 70: 908-10.

6. Safety and performance of the Lithoplasty ${ }^{\circledR}$ Technology System [Internet]. Fremont (CA): Shockwave Medical Inc; 2017. Available from: http://shockwavemedical. com/intl/clinical-data/disrupt-pad-i/.

7. Gorla R, Cannone GS, Bedogni F, De Marco F. Transfemoral aortic valve implantation following lithoplasty of iliac artery in a patient with poor vascular access. Catheter Cardiovasc Interv 2018; doi: 10.1002/ccd.27812. 